WM'03 Conference, February 23-27, 2003, Tucson, AZ

\title{
DIAMOND WIRE CUTTING OF THE TOKAMAK FUSION TEST REACTOR
}

\author{
Keith Rule \\ Princeton Plasma Physics Laboratory \\ Erik Perry and Robert Parsells \\ Princeton Plasma Physics Laboratory
}

\begin{abstract}
The Tokamak Fusion Test Reactor (TFTR) is a one-of-a-kind, tritium-fueled fusion research reactor that ceased operation in April 1997. As a result, decommissioning commenced in October 1999. The 100 cubic meter volume of the donut-shaped reactor makes it the second largest fusion reactor in the world. The deuterium-tritium experiments resulted in contaminating the vacuum vessel with tritium and activating the materials with $14 \mathrm{Mev}$ neutrons. The total tritium content within the vessel is in excess of 7,000 Curies while dose rates approach 50 $\mathrm{mRem} / \mathrm{hr}$. These radiological hazards along with the size of the Tokamak present a unique and challenging task for dismantling.

Engineers at the Princeton Plasma Physics Laboratory (PPPL) decided to investigate an alternate, innovative approach for dismantlement of the TFTR vacuum vessel: diamond wire cutting technology. In August 1999, this technology was successfully demonstrated and evaluated on vacuum vessel surrogates. Subsequently, the technology was improved and redesigned for the actual cutting of the vacuum vessel. 10 complete cuts were performed in a 6month period to complete the removal of this unprecedented type of D\&D activity.
\end{abstract}

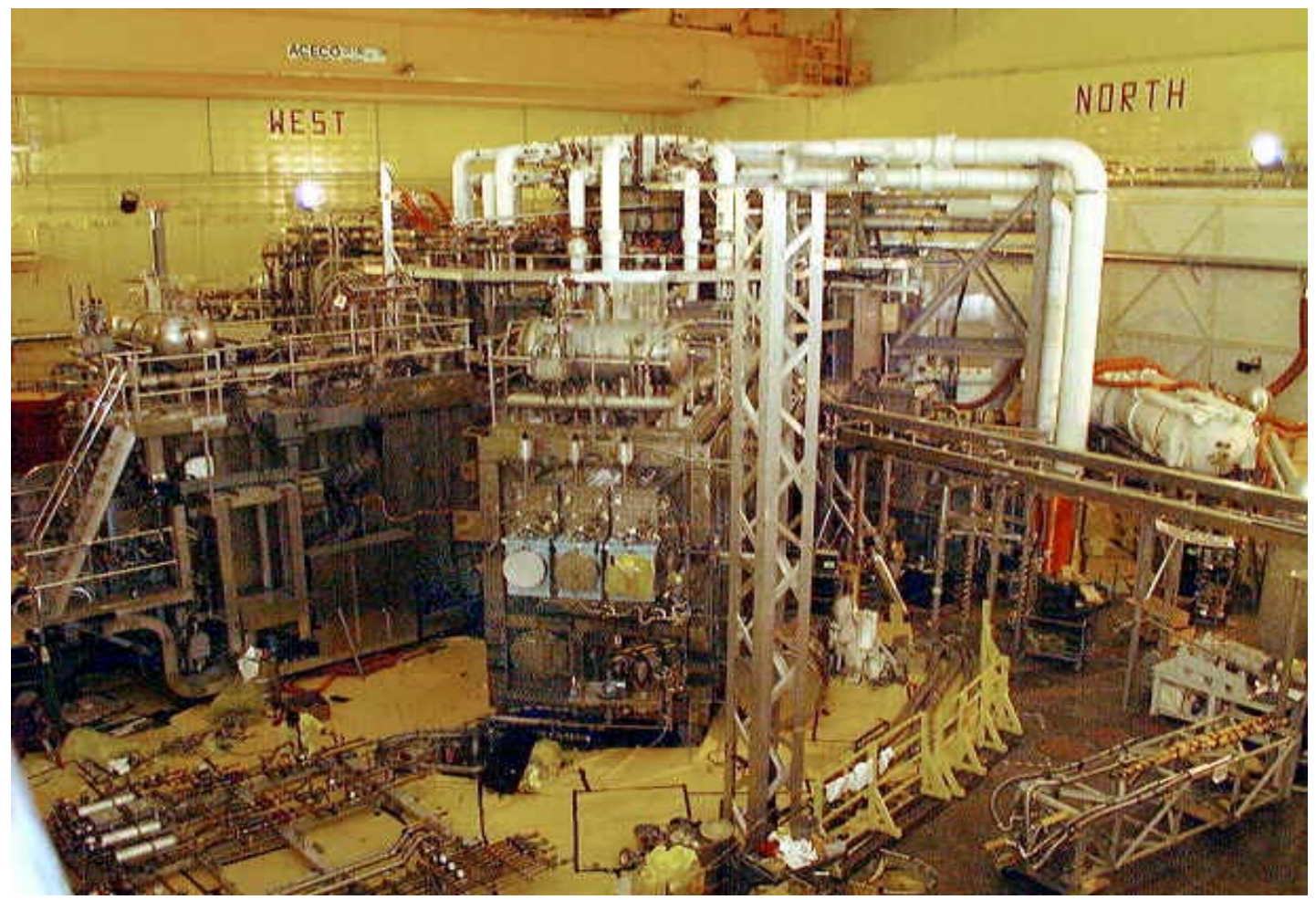

Figure 1. Photo of TFTR initial phase of D\&D - 1999 


\section{WHAT IS DIAMOND WIRE CUTTING?}

The diamond wire cutting process, originally developed for marble and granite mining operations in South Africa and Europe, has existed for more than 25 years. When mining operators and quarries demanded safer, cheaper, more productive cutting products, several manufacturers in Europe developed diamond wire for nondestructive rock extraction. Diamond wire cutting is a revolutionary method of precision-cutting dimensional stone. Diamondimpregnated beads, fitted to a continuous strand of spring or rubber-encapsulated steel wire, are looped into the drive pulley of the wire sawing machine and driven at high speed through stone to make precise horizontal or vertical cuts. With its fast, clean cutting action, wire sawing has proven to be an extremely efficient system for nondestructive extraction and demolition mining operations. Born in the world's quarries, diamond wire has become a prerequisite for improving recovery rates in stone extraction. (1)

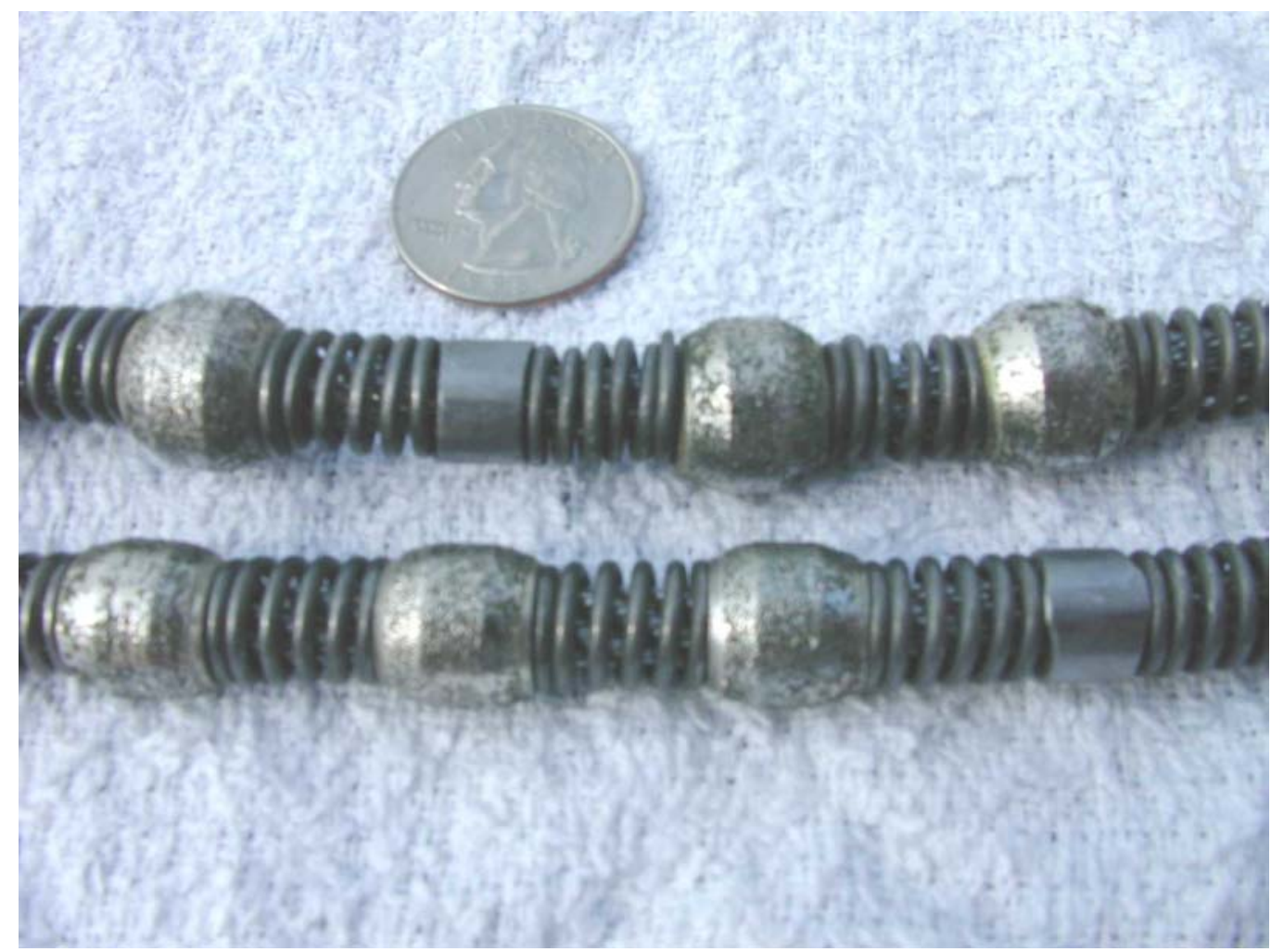

Figure 2 - Photo of Diamond Wire

Diamond wires are now used throughout the world to cut marble, granite, sandstone, steel pipes, and reinforced concrete. Several types of wire configurations and beads are available from a number of worldwide producers for specific applications. The industry's improvements to this technology has led to its expanded application to underwater cutting of piping, concrete and metal structures, and other types of stone and precious materials. Diamond wire and diamond wire saws can cut most materials. As a result, this technology has been used on several decommissioning projects, including the dismantling of the concrete support structures and shielding at the Fort St. Vrain and Shoreham nuclear power plants. These structures contained steel plate and other steel reinforcement that was cut as part of the process. Encouraged by these successful projects, PPPL decided to research and develop applications of the technology for the segmentation of the TFTR vacuum vessel. 


\section{THE STANDARD TECHNOLOGY}

The baseline planning for dismantling the vacuum vessel utilized plasma-arc torch cutting. This involves personnel wearing air-supplied bubble suits entering the vessel to access and remove internal components. Some components could be removed by mechanical means, while others required plasma cutting. The use of the plasma torch would have required additional specialized equipment, necessitating dedicated engineering and specialized fabrication of remote/semi-remote cutting devices. Also, using the plasma-arc torch would have resulted in the release of radioactive airborne contamination and harmful gases, requiring extensive containment, filtering, and respiratory protection. The health and safety of personnel working within the confined space in air-supplied hoods, alongside heat-generating cutting equipment, was of great concern, and such work was considered to be a high-risk operation.

At least 30 entries into the vacuum vessel would have been necessary to systematically set up and remove multiple layers of obstructions prior to cutting the vacuum vessel stainless steel shell. These materials consisted of precision-mounted graphite tiles, stainless steel protective plates, INCONEL ${ }^{\oplus}$ support plates, and various ceramic components. Most of these structures had been manufactured and installed with strict tolerance, further complicating and increasing the difficulty of manual removal, especially because of the need for multiple layers of protective clothing. This leads to increased radiological exposure along with higher risk of occupational safety hazards.

Diamond wire cutting, on the other hand, can be performed remotely from outside the vacuum vessel, completely removing many of the health and safety concerns associated with plasma cutting.

\section{RESEARCH AND DEVELOPMENT}

There were four main questions to be considered for the successful application of diamond wire cutting technology to cut the complex TFTR vacuum vessel structure:

What types of cooling media would give the best cutting performance while providing for proper control of the radionuclide emissions?

What remote operating and control methods could be developed to control the direction and angle of the wire for optimal cutting and reduced wire failure?

What controls would be necessary to eliminate the release of radioactivity outside of the cutting boundaries?

Would this technology cut through the complex metal structure (with INCONEL plating) in a more cost-effective and safe manner than the baseline technology?

Vacuum vessel surrogates were fabricated, each consisting of a cylinder approximately 48 in. long and $90 \mathrm{in.} \mathrm{high} \mathrm{(diameter),} \mathrm{the} \mathrm{same} \mathrm{dimensions} \mathrm{and} \mathrm{shape} \mathrm{as} \mathrm{the} \mathrm{real} \mathrm{segments.} \mathrm{A}$ test bed was designed and constructed to support and elevate the surrogates during trial cuttings. The surrogate consisted of a one half-in. Type 304 stainless steel shell with internal layers of 5/8in. INCONEL plate, a 1-in. Type 304 stainless steel plate, and 2 in. of graphite block.

\section{Cooling with Cryogenics}

Both water and cryogenic cooling were considered for the cutting tests. Cryogenic cooling had been used for diamond wire cutting at other facilities with limited success. Water is an inexpensive cooling medium, it can be reclaimed and reused, and it controls/suppresses the generation of dust. However, controlling the spray/mist, preventing waste migration, and collecting the water would be challenging. 
WM'03 Conference, February 23-27, 2003, Tucson, AZ

A cryogenic cooling medium is more expensive than water. It cannot be reused and would be expended into the area where the cutting is taking place. In addition, workers face the prospect of dust control. On the other hand, cryogenics provides cooler cutting temperatures, which may improve wire performance and clean the wire and diamond beads. Cryogenic cooling was ultimately selected.

\section{Remote Control Systems}

Diamond wire cutting is performed remotely after the initial setup of the wire and hydraulic control systems. A hydraulic motor drives the main drive saw, which is aligned with the direction of the cut. The wire is wrapped around the drive wheel and object to be cut. An electro-hydraulic power unit drives the saw and pros force on the wire. This power unit can be placed a minimum of 25 feet from the cutting location, depending on the length of hydraulic hose selected. This arrangement minimizes the risk of injury.

To successfully cut the complex TFTR vacuum chamber safely at the lowest cost while maximizing worker health and safety, several devices and controls had to be developed. An automated pulley system was designed and installed to control the approach angle of the diamond wire to the vacuum vessel. The approach angle was critical to proper cutting while eliminating the possibility of wire failure due to the extremely sharp edges created when cutting the various layers of metals. The pulleys were mounted on a control shaft and moved by remote control using servomotors. Figures 3 and 4 depict the orientation of the system and the various pulley locations (travel) that were available. The design provided for two types of cutting approaches. The more common approach of pulling the wire toward the saw is depicted in Fig. 3. Figure 4 depicts what we refer to as a "push cut," in that the wire cuts into the object and moves away from the saw. This is much like a band saw.

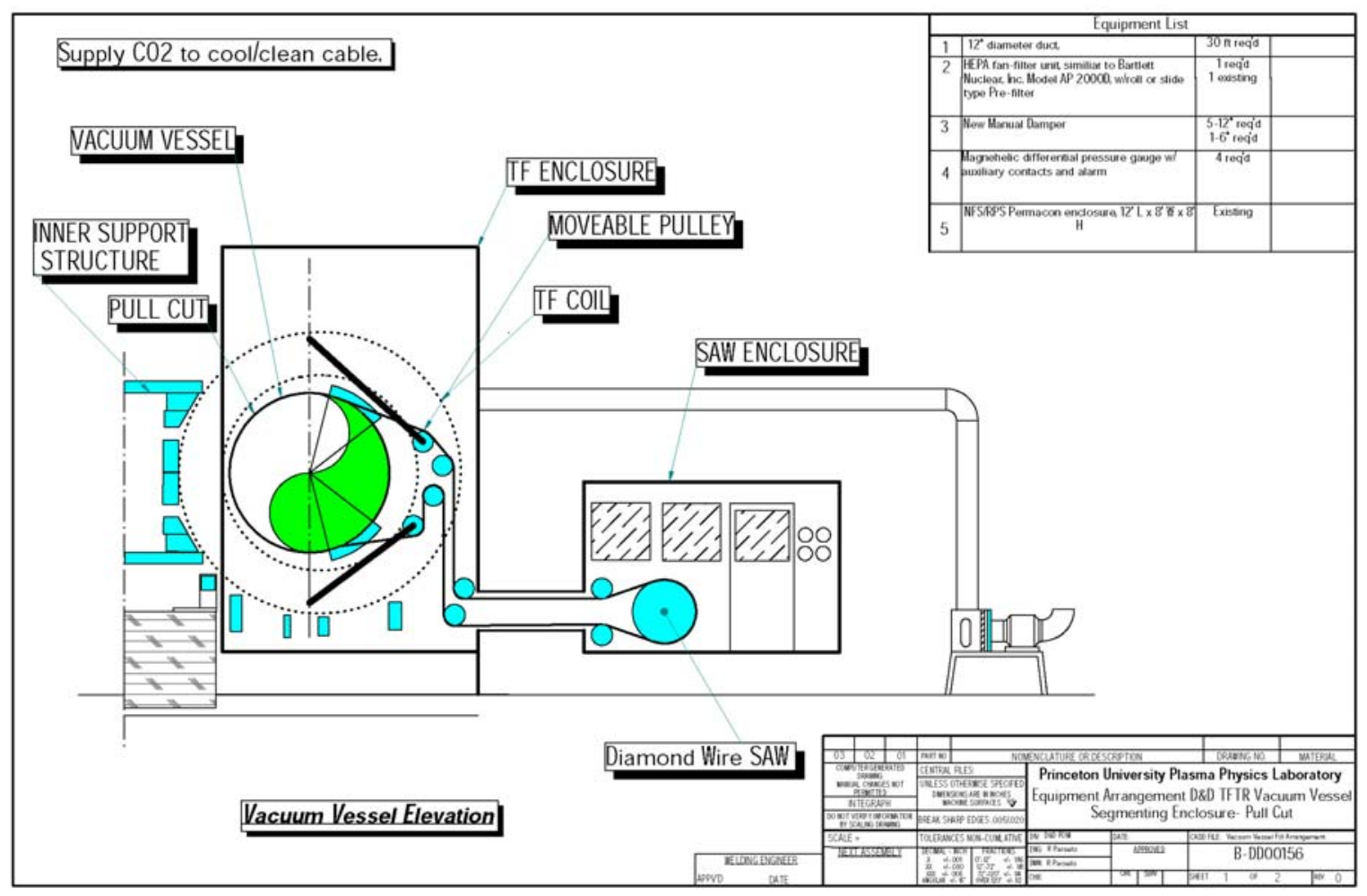

Figure 3 - Pull Cut 
WM'03 Conference, February 23-27, 2003, Tucson, AZ

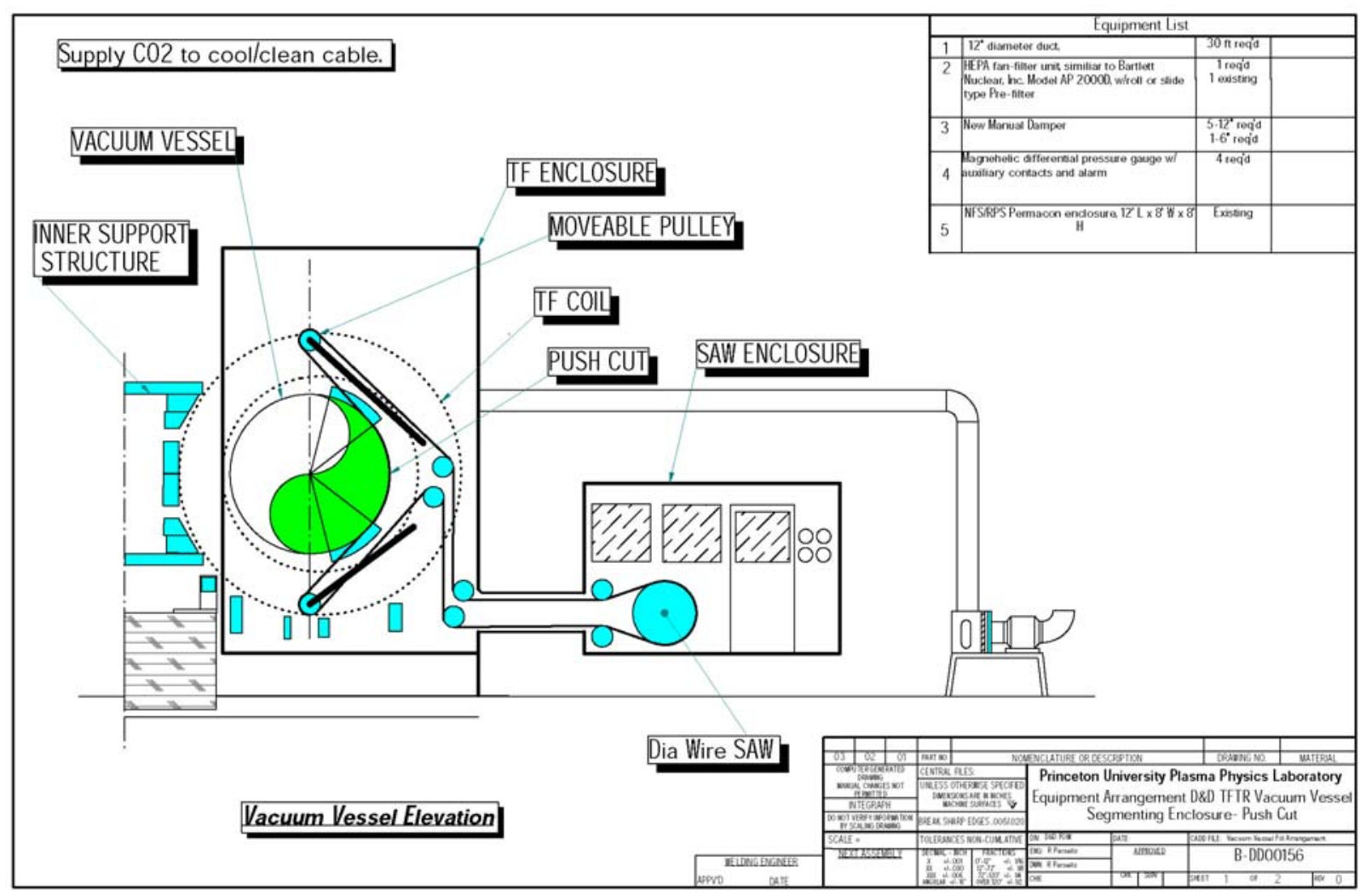

Figure 4 - Push Cut

The more common "pull-cut" approach was used to begin cutting operations and was typically used for 60 percent of the total cutting area. The less common approach of pushing the wire into the vacuum vessel was used to complete the severing and was quite successful. On several occasions the wire cutting became less effective or bound in the kerf during a pull cut. The use of the push cut allowed us to relieve stresses and complete the cut through the entire 90in.-diameter vessel.

\section{Engineering Controls}

Once the cutting and control techniques were defined, containment and contamination control became paramount. These were accomplished utilizing three techniques. The first was to fill the vacuum vessel with a low-density concrete. The second was to control particulate emissions directly around the wire travel. The third was to provide containment and ventilation around the cutting operation.

The product Geocell foamed lightweight concrete was selected as the low-density concrete. The effect of the concrete on the graphite tiles and its ability to contain tritium was evaluated in a detailed study (2). The concrete proved to be quite effective in trapping tritium and preventing off-gassing. It also contributed two other benefits: additional shielding from gamma radiation and the elimination of the void of the inner volume for burial requirements.

A ventilation shroud was designed and installed around the path of wire travel. Slots were furnished for the various angles the wire would travel along this path. Connections were arranged for a $3000 \mathrm{ft} / \mathrm{min}$ high-efficiency dust collection system to provide suction on this shroud and discharge back to the main containment. An automated system maintained filter cleanliness and collected the particulates in a 55-gallon drum. This system collected the majority of the dust generated by the cutting. 


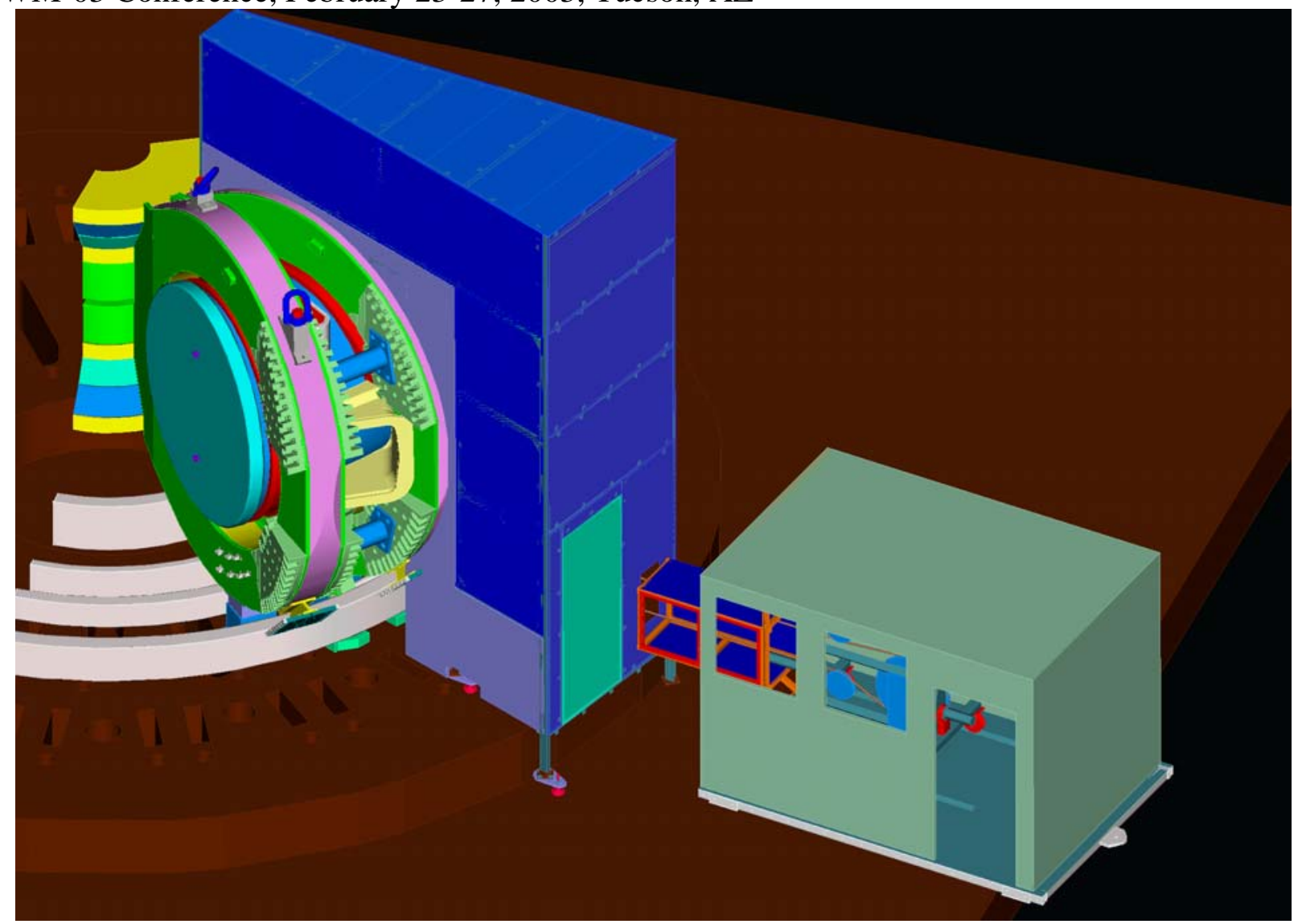

Figure 5 - Diamond Wire Cutting Arrangement

The vacuum vessel, dust collection assembly, and pulley system were contained within a Lexan and Unistrut rigid containment shroud, completely sealed to all surfaces and ventilated to our stack using a $2000 \mathrm{ft} / \mathrm{min}$ high-efficiency particulate (HEPA) ventilation system. The diamond wire saw was contained within a Permacon containment, which was connected to the main containment allowing for both to be ventilated with the same HEPA system. The combination of the two ventilation systems achieved the objective of containing the tritium and radioactive particulates. Consequently, we were able to control emissions and conduct the cutting with minimal worker entry into the cutting area.

\section{IS THE TECHNOLOGY MORE EFFECTIVE AND SAFER?}

During the R\&D phase it became clear that this technology would perform the cutting successfully and in a safe manner. Weeks of testing took place to train operators, define operating parameters, and refine our process and procedures for the actual cutting on the radioactive vacuum vessel. Techniques to improve wire crimping allowed us to perform cutting without wire failures. As one would expect, this is quite important. If a wire breaks, personnel must enter the containment to repair the wire. This involves the use of protective clothing and can require the use of respirators.

The automated pulley system further improved the cutting and also prevented wire failure. Operating experience was gained by adjusting the pulley positions to minimize the possibility of the sharp layers of metal from catching or cutting parts of the diamond wire.

The particulate collection system and assembly maintained a relatively clean environment within the containment. The system worked well enough to eliminate visible dust (during cutting) when combined with the HEPA ventilation system and significantly lowered surface contamination levels within the main containment. More importantly, both of these systems prevented any release of contamination outside the cutting area into the room. 


\section{SUCCESS}

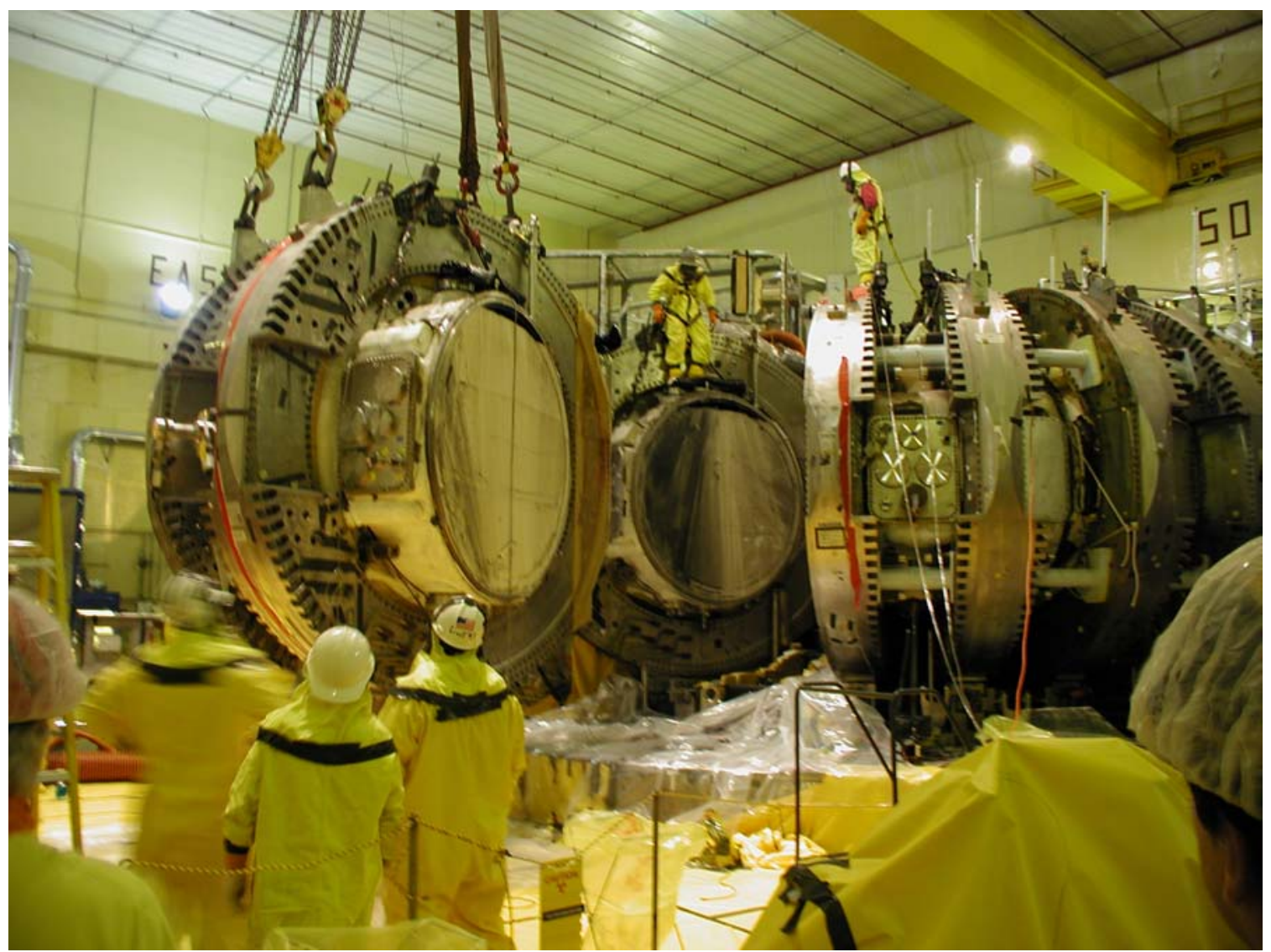

Figure 6-Removal of First Segment

The process implementation began in June 2001 with the filling of the vacuum vessel with the Geocell concrete. The concrete was pumped in three successive events with at least 24 hours of cure time between lifts. A total of 100 cubic yards of concrete was added with an average density of $50 \mathrm{lb} / \mathrm{ft}^{3}$. This completed the first phase of containment and contamination control.

The automated pulley system was installed at the selected cut location along with the particulate collection shrouds and apparatus. Several pulleys were mounted and positioned to transition the diamond wire from the automated pulley system to the saw. With these systems in place, the main containment and saw containment were installed, sealed, and joined. The cryogenic cooling system was strategically positioned to cool and clean the diamond wire. An 11-mm-diameter diamond wire was used to begin and perform most of the cutting. When a new wire needed to be installed, because of smaller kerfs due to bead wear, a 10-mm wire was sometimes needed.

The first of 10 cuts began in late August 2001; all cuts were completed in late February 2002. The average cutting time to completely sever the segment was 15 hours, with all cutting taking place without personnel in the containment. All 10 cuts were performed as planned. The "pull cut" method was utilized for the majority of cutting, while the "push cut" was used to complete the cut and free the segment. While there were instances of wire failure due to binding of the wire from stresses and fixtures within the vacuum vessel, a minimal number of entries into the containment were necessary to correct these conditions.

The contamination control systems, procedures, and radiation protection practices complemented the engineering controls to prevent the spread of contamination beyond the 
planned control areas and containments. No unplanned personnel exposures occurred, and all cutting was performed without any occupational injury.

\section{RE-ENGINEERING A MATURE TECHNOLOGY}

Diamond wire cutting is a mature technology for concrete cutting applications. As a result of this R\&D and the subsequent successful cutting, the technology has also proven to be applicable to size-reduction of large metal vessels such as reactors, heat exchangers, and tanks, when combined with some form of concrete matrix. The technology is particularly advantageous when there are significant health and safety concerns. Skilled and experienced machinists are critical to the successful cutting and performance. Selection of a concrete filler that meets the cutting, stabilization, and shipping constraints is also critical to the success of the project.

Strong consideration must be given to the selection of water or cryogenics as the cooling medium. This decision must take into account particular radionuclide hazards and the availability of feasible engineering controls for each medium. Liquid waste generation and subsequent solidification and treatment can be costly and difficult. In contrast, the airborne dry particulate generated when using cryogenics is a concern along with oxygen deficiency.

Finally, involving all trade personnel in the planning, testing, and construction phases is also necessary to the success of the technology and will result in fewer modifications and lost time during the actual implementation.

\section{FUTURE APPLICATIONS}

We have successfully demonstrated the use of diamond wire cutting on large metal structures composed of soft metals, such as stainless steel, and one of the hardest metals, INCONEL, in conjunction with a concrete-matrix. In addition, this technology is particularly advantageous when compared to the significant health and safety concerns associated with the baseline technology. Lighter weight concrete, such as Geocell, increased the capability of the technology, while affording radionuclide stabilization, strength, and shielding. Diamond wire cutting has proved superior to plasma-arc cutting for large metal vessels and heat exchangers and is especially beneficial where there are significant radiological hazards. The technology provided significant improvements in worker safety and cutting performance compared to the baseline.

\section{ACKNOWLEDGMENTS}

The authors would like to acknowledge the financial and management support provided by the D\&D Focus Area team at Federal Energy Technology Center in Morgantown, W.V., and in particular, Jerry Hyde and Steve Bossart from that center. This work is also supported by the US. DOE under contract DE-AC02-76CH03073 and managed by the local Princeton Group representatives, Wm. Jerry Faul, George Cava and Jeffrey Makiel.

This paper is Reprinted with permission form the May/June 2002 issue from Radwaste Solutions (C) 2002 by American Nuclear Society.

\section{REFERENCES}

1. www.boartlongyear.com

2. P. LaMarch, et al., "Tritium Considerations in the Segmentation of the TFTR Vacuum Vessel," presented at $19^{\text {th }}$ Symp. Fusion Energy, Atlantic City, New Jersey, January 2002. 